\title{
Effects of selenoprotein $P$ on the contraction and relaxation of the airway smooth muscle
}

\author{
Sachie K. Ogawa ${ }^{1}$, Min-Chul Shin ${ }^{1}$, Masaki Hirashima ${ }^{2}$, Norio Akaike ${ }^{1}$ and Yushi Ito ${ }^{1}$ \\ ${ }^{1}$ Research Division for Life Sciences, Kumamoto Health Science University, 325 Izumi-machi, Kumamoto 861-5598, Japan \\ ${ }^{2}$ Division 1 Research Department, The Chemo-Sero-Therapeutic Research Institute, 1314-1 Kyokushin Kawabe, Kikuchi, \\ Kumamoto 869-1298, Japan
}

\begin{abstract}
Selenoprotein $\mathrm{P}(\mathrm{SeP})$ not only represents the major selenoprotein in plasma, but also provides more than $50 \%$ of the total plasma selenium. However, there is no report concerning the direct action of selenium or selenium-containing compounds on the contraction and relaxation of the airway smooth muscle. Therefore, we investigated the effects of SeP and sodium selenite (SS) on the indirectly induced contraction and relaxation of the cat bronchi, and gel contraction of cultured bovine tracheal smooth muscle cells (BTSMC) induced by ATP. In the present results, SeP or SS suppressed the amplitude of twitch-like contractions of cat bronchiole without affecting the nonadrenergic and non-cholinergic (NANC) relaxations evoked by electrical field stimulation. SeP also suppressed the ATP-induced gel contraction of BTSMC. These results suggest that SeP suppresses the amplitude of twitch-like contraction of cat bronchiole by acting directly on the bronchiolar smooth muscle.
\end{abstract}

Key words: Selenoprotein P — Sodium selenite — Bovine tracheal smooth muscle

\section{Introduction}

Selenium was first confirmed as toxic agent in the livestock that consumed selenium accumulator plants in 1933 (see for example, Schwarz and Foltz 1999). Then, selenium was identified as an essential nutrient for laboratory animals, and essentiality for mammalian species was established in 1973 with the discovery that enzyme glutathione peroxidase contains selenium (Rotruck et al. 1973). Thus, selenium is now recognized as an essential trace element in the mammalian diet that is an antioxidant important for many aspects of human health (Rayman 2000). For instance, it has been shown that selenium-deficiency causes many diseases including Keshan disease, a dilative cardiomyopathy primarily affecting children (Ge et al. 1983), coronary heart disease (Salonen et al. 1982) and cancers of large intestine, rectum, prostate, breast, ovary and lung (Schrauzer et al. 1977). Sodium selenite (SS) is commonly used as a dietary supplement for the treatment of selenium deficiency (Neve 1991). However,

Correspondence to: Yushi Ito, Research Division for Life Sciences, Kumamoto Health Science University, 325 Izumi-machi, Kumamoto 861-5598, Japan

E-mail: itou@kumamoto-hsu.ac.jp it should be recalled that selenium containing compounds are toxic and that therapeutic index - the difference between beneficial and toxic effects - is rather small (for example, see Gromer et al. 2005).

It has been shown that experimental chronic selenium toxicity in animals affect the major organs including liver, spleen, heart, kidneys and pancreas; more particularly SS has been shown to cause cellular dysfunction in a number of tissues including erythrocytes (Young et al. 1981), hepatocytes (Anundi et al. 1982) and skeletal muscle (Lin-Shiau et al. 1989). In the cardiac muscle, it was reported that the administration of SS causes a positive inotropic effect which appeared even after the blockade of adrenergic receptors and in segments perfused with a Krebs-bicarbonate solution that was deficient in oxygen (Aviado et al. 1975).

In the lung, oxidant stress results in inflammation and tissue damage in the respiratory system, and later in immune damage, and those individuals with lowered cellular reducing capacity are at increased risk to develop asthma (Greene 1995). On the other hand, there are reports that intratracheal deposition of excessive SS results in pulmonary dysfunction and cytotoxicity in guinea pigs (Bell et al. 1997), and acute intratracheal selenium dioxide exposure decreases respiratory rate and increases lung resistance 
(Nonavinakere et al. 1999). In addition, descriptive studies in humans investigating the relationship between selenium and asthma have presented inconsistent results. Namely, the concept of low selenium status as a potential risk factor for increased prevalence or severity of asthma is based on the role that selenium, through its incorporation, plays as a potent antioxidant capable of augmenting the oxidative stress that accompanies asthma. However, selenium intake also exerts a significant influence over immune responses through regulation of $\mathrm{T}$ helper 2 responses that drive allergic asthma. Thus, selenium has dual effects on controlling oxidative stress in the lung and regulating $\mathrm{T}$ helper 2 responses (Hoffmann 2008). Furthermore, it was reported that selenium has a regulatory role in the balance between the muscarinic receptor-mediated contraction and $\beta$ adrenergic-mediated relaxation of the pulmonary smooth muscle (Doelman and Bast 1990). Till now, however, there is no report concerning the direct action of selenium or selenium containing compounds on the contraction and relaxation of the airway smooth muscle. Selenoprotein $\mathrm{P}$ (SeP) not only represents the major selenoprotein in plasma, but also provides more than $50 \%$ of the total plasma selenium. Therefore in the present study, we observed the effects of SeP and SS on the indirectly induced contraction and relaxation of the cat bronchi, and ATP-induced gel contraction of cultured bovine tracheal smooth muscle cells (BTSMC).

\section{Material and Methods}

\section{Tissue preparations}

All experiments were performed in accordance with the guiding principles for care and use of animals in the field of physiological sciences of the Physiological Society of Japan and were approved by the Animal Ethics Committee of Kumamoto Health Science University. Adult mongrel cats of either sex (2-3 kg) were anaesthetized with sodium pentobarbitone $(30-40 \mathrm{mg} / \mathrm{kg}$, i.p.) and then bled. The trachea and whole pulmonary lobes were quickly resected from the main bronchus and placed in modified Krebs solution of the following composition (mM): $\mathrm{Na}^{+} 137.4, \mathrm{~K}^{+} 5.9$, $\mathrm{Mg}^{2+} 1.2, \mathrm{Ca}^{2+}$ 2.5, $\mathrm{Cl}^{-} 134.0, \mathrm{H}_{2} \mathrm{PO}_{4}^{-}{ }^{-} 1.2, \mathrm{HCO}_{3}{ }^{-} 15.5$ and glucose 11.5 . The solution was aerated with $97 \% \mathrm{O}_{2}$ and $3 \%$ $\mathrm{CO}_{2}$ and the $\mathrm{pH}$ was 7.3-7.4. Small airways were carefully excised from the lung tissue and lung parenchyma, and pulmonary vessels running along the bronchiolar branch were carefully removed under microscopic observation. Since the branching pattern of the cat airways is not symmetrical and regular as in humans (Mortensen et al. 1983) and is similar to that of dogs (Amis and McKiernan 1986), the diameter of the bronchi does not necessarily correlate with their order of branching, as described earlier (Takahashi et al. 1995). Segments of isolated bronchioles (0.5-2 mm O.D.) were cut into rings $2-3 \mathrm{~mm}$ wide. As much airway epithelium as possible was carefully removed by mechanical rubbing according to the method described earlier (Xie et al. 1991), since it is known that electric current (Xie et al. 1991) can release factors from the airway epithelial cells which induce relaxation.

\section{Mechanical recording}

For measurement of mechanical responses, the ring preparations of bronchioles were hooked horizontally by a pair of right-angled fine needles in a $1 \mathrm{ml}$ organ bath, through which the test solution flowed continuously at a rate of $2 \mathrm{ml} / \mathrm{min}$. One needle was fixed to the wall of the chamber, the other was connected to a manipulator and to an isometric mechano-transducer (RCA-5734, Nihon Kohden) through a $1 \mathrm{~mm}$-wide slit made in the other wall of the chamber (Takahashi et al. 1995). The strips were set up with an initial tension of 1-2 $\mathrm{mN}$ and mechanical activity was recorded with a pen recorder. Electrical field stimulation (EFS, 10 or 30 pulses of $0.5 \mathrm{~ms}$ duration at $20 \mathrm{~Hz}$ ) was applied through a pair of Ag-AgCl plates fixed to both sides of the inner surface of the chamber, so that current pulse would pass transversely across the ring preparations of the bronchioles.

Twitch-like contractions in response to EFS were recorded in the presence of guanethidine, indomethacin, $\mathrm{L}-\mathrm{N}^{\omega}$-nitroarginine methylester (L-NAME) (10 $\mu \mathrm{M}$ each) and rabbit antiporcine VIP antiserum $(1 \mu \mathrm{g} / \mathrm{ml})$ due to the following reasons. Namely, it is known that EFS applied to the tracheal or bronchiolar tissue evokes propronolol-sensitive muscle relaxation (Ito and Tajima 1981) and EFS also stimulates the synthesis of prostaglandins which suppresses the amplitude of twitch-like contraction of dog trachea (Ito and Tajima 1981) or bronchiole (Inoue and Ito 1986), and cat trachea (Hakoda and Ito 1990). Furthermore, EFS stimulates non-adrenergic and non-cholinergic (NANC) inhibitory nerves in the cat bronchial tissue and release NANC neurotransmitters, which affect the muscle tone and amplitude of twitch-like contractions. Although the neurotransmitters responsible for the NANC relaxation of the cat airway has not been conclusively identified, we have demonstrated that at least two substances, nitric oxide (NO) or NO-containing compounds for the fast relaxation and vasoactive intestinal polypeptide (VIP) or VIP-like peptide for slow relaxation are involved (Matsuzaki et al. 1980; Palmer et al. 1986; Hakoda et al. 1991; Takahashi et al. 1995; Tanaka et al. 1996). Therefore, to record twitch-like contractions with stable amplitude, L-NAME and VIP-antiserum were added to the modified Krebs solution in the presence of guanethidine and indomethacin $(1 \mu \mathrm{M}$ each). 


\section{Cell culture}

Tracheas of 1-year-old calves were obtained from a local slaughterhouse. Smooth muscle cells were dissociated from the tracheas and cultured in Dulbecco's modified Eagle's medium (DMEM; Gibco, Invitrogen Life Technologies, Paisley, UK) with $10 \%$ fetal bovine serum (FBS; Invitrogen). Confluent cells were harvested following trypsin digestion and stored at $-80^{\circ} \mathrm{C}$, and $2 \mathrm{nd}$ or $3 \mathrm{rd}$ subcultured cells were used for this study (Sakai et al. 2003). The present study was performed on BTSMC obtained from three tracheas.

\section{Gel contraction assay}

A gel contraction assay (Sakai et al. 2003) was used to measure the contraction or relaxation of BTSMC. Stored BTSMC were resuspended in $0.5 \mathrm{ml}$ solution containing $0.2 \%$ type IA collagen (Nitta Gelatin Inc., Osaka, Japan) in DMEM and poured onto 24 -well culture plates at a density of $2.5 \times 10^{5}$ cells/well for $10 \mathrm{~min}$ at $37^{\circ} \mathrm{C}$ until a gel formed. Then $1 \mathrm{ml}$ of DMEM with $10 \%$ FBS was added to the gel. After culturing for 3 days at $37^{\circ} \mathrm{C}$, the collagen gel containing BTSMC was used for the contraction assay. The BTSMC embedded in the gel were randomly oriented, and had a spindle-like shape (Fig. 3A). The number of embedded cells did not increase significantly in 3 days. Before each experiment, DMEM with 10\% FBS was replaced by Krebs solution, and the lateral surface of the gel was carefully detached from the culture well with a fine needle. The culture plate was then placed on a hotplate (ND-1, As One, Osaka, Japan) and kept $37^{\circ} \mathrm{C}$ during the experiment. The gel images were captured with a digital camera (Coolpix S4, Tokyo, Japan) every 1 min throughout the experiment. The area of the gel surface was evaluated by measuring the pixel numbers of the gel surface with image analysis software (Adobe Photoshop, Adobe Systems, Inc., USA). The effects of each drug were quantified as changes in gel surface area. Surface area measurements of each gel were made pre- and post-drug application. Because the degree of contraction in control gels (without drug treatment) varied between batches, the data of drug-treated groups were calculated as a relative value compared with the data of the drug-untreated groups obtained in the same batch.

\section{Purification of full-length SeP}

Purification of SeP from plasma was performed according to the method described elsewhere (Saito et al. 1999). Briefly, human fresh lyophilized plasma (2 1) was fully thawed in a warm bath and then placed in a cooling chamber. To the plasma was added 100 g PEG 4000 in portions while stirring. After completion of addition, stirring was further continued for 1 hour. The mixture was centrifuged at 10,000 $\times g$ for 20 minutes. Supernatant was recovered and filtered through AP25 (Millipore). A total of the filtrate was applied to a column charged with $100 \mathrm{ml}$ heparin Sepharose (Amersham Pharmacia Biotech) previously equilibrated with $20 \mathrm{mM}$ phosphate buffer (20 mM phosphate, $\mathrm{pH} 7.4$, $0.15 \mathrm{M} \mathrm{NaCl}, 0.2 \mathrm{mM}$ EDTA). After washing the column, adsorbed proteins were gradient eluted with a gradient of a salt concentration ranging from $0.15 \mathrm{M}$ to $0.6 \mathrm{M}$. Each $5 \mathrm{ml}$ of eluted fractions were taken, an amount of SeP contained in each fraction was determined by ELISA and fractions containing SeP were pooled. In order to avoid fragmentation of SeP during the purification procedure, diisopropylfluorophosphate (DFP) was added at a final concentration of $5 \mathrm{mM}$. The fraction was then diluted 6folds with $20 \mathrm{mM}$ Tris buffer (20 mM Tris- $\mathrm{HCl}, \mathrm{pH} 8)$. At this stage, a total amount of the sample was 0.75 to 1 liter. The sample was applied to $40 \mathrm{ml}$ Q sepharose (Amersham
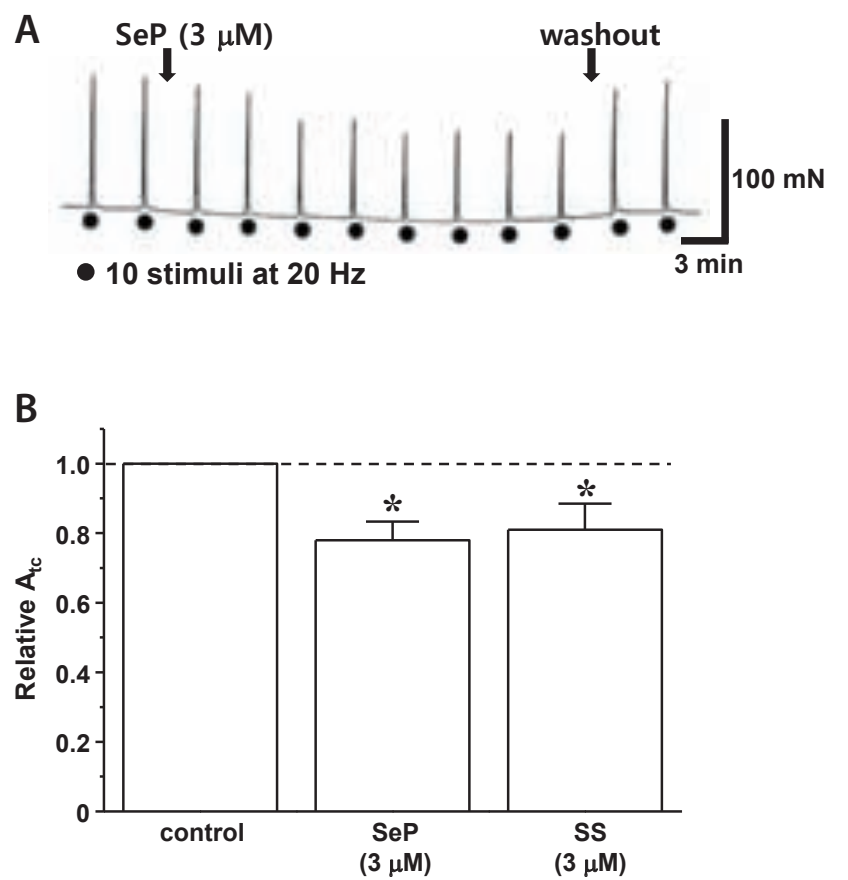

Figure 1. Effects of SeP $(3 \mu \mathrm{M})$ on the twitch-like contraction of cat bronchiole evoked by EFS in the presence of guanethidine, indomethacin, L-NAME (10 $\mu \mathrm{M}$ each $)$ and VIP-antiserum $(1 \mu \mathrm{g} /$ $\mathrm{ml})$. A. Actual trace of the effects of $\operatorname{SeP}(3 \mu \mathrm{M})$ on the twitch-like contractions evoked by EFS (10 stimuli at $20 \mathrm{~Hz}$ ). B. Summary of the effects of SeP and SS ( $3 \mu \mathrm{M}$ each) on the amplitude of twitch-like contraction: 16 or 7 bronchiolar preparations were excised from 5 or 3 cats, respectively. SeP, selenoprotein P; SS, sodium selenite; Relative Atc, relative amplitude of twitch-like contraction; ${ }^{*} p<0.05$. 
Pharmacia Biotech) column equilibrated with $20 \mathrm{mM}$ Tris buffer. The column was washed with 250 to $300 \mathrm{ml}$ equilibration buffer and elution was performed with a gradient of a salt concentration up to $0.25 \mathrm{M}$ using $250 \mathrm{ml}$ buffer. The obtained fractions were determined by ELISA and fractions containing $\mathrm{SeP}$ were pooled. To this pooled fraction was added diisopropylfluorophosphate at final concentration of $5 \mathrm{mM}$.

To the obtained pooled fraction was then added imidazole at a final concentration of $2 \mathrm{mM}$ and the mixture was applied to $4 \mathrm{ml} \mathrm{Ni-NTA}$ agarose gel (Qiagen) column. The column has previously been equilibrated with $20 \mathrm{mM}$ Tris buffer (20 mM Tris-HCl, pH 8.2 mM imidazole). After application, the column was washed with $30 \mathrm{ml}$ washing buffer (20 mM Tris- $\mathrm{HCl}, \mathrm{pH}$ 8, $20 \mathrm{mM}$ Imidazole, $1 \mathrm{M} \mathrm{Na}$ $\mathrm{Cl}$ ) and elution was performed with an elution buffer (20 mM Tris- $\mathrm{HCl}, \mathrm{pH}$ 8, $150 \mathrm{mM}$ imidazole, $1 \mathrm{M} \mathrm{NaCl}$ ). Each $1 \mathrm{ml}$ of eluted fractions was taken and fractions were pooled where peaked absorbance was observed at O.D. $280 \mathrm{~nm}$. This fraction was concentrated with centrifugation to $2.5 \mathrm{ml}$ with SpeedVac (Savant Speed Vac). Finally, the concentrated SeP fraction was applied to PD-10 column (Amersham Pharmacia Biotech) equilibrated with a phosphate buffer, followed by desalting and exchange of a buffer. SeP thus obtained showed migration of M.W. around 67,000 in SDS-PAGE, most of which was thought to be full-length SeP.

\section{Chemicals and data analysis}

The following drugs were used: $\mathrm{N}^{\omega}$-nitro-L-Arginine methyl ester (L-NAME) (Nakarai Chemicals), atropine sulfate, guanethidinemonosulfate 5-hydroxytryptamine creatinine sulfate (5-HT), acetylcholine chloride, nifedipine and tetrodotoxin (TTX) (Sigma), $\omega$-CgTX GVIA, $\omega$-CgTX MVIIC, $\omega$-agatoxin IVA and nimodipine (Calbiochem), rabbit antiporcine VIP antiserum (Peptide Institute, Osaka Japan) and s-nitroso-N-acetyl penicillamine (SNAP) (Dojin). Except nifedipine and nimodipine which were dissolved in DMSO, all drugs were dissolved in distilled water.

The mechanical recordings were carried out at $35-36^{\circ} \mathrm{C}$. Results (amplitude of twitch-like contraction and NANC relaxation) are expressed as mean \pm S.E. and were analyzed for significance by Student's $t$-test.

To analyze the effect of SeP $(3 \mu \mathrm{M})$ on ATP-induced contraction of the gel embedded BTSMC, SeP was applied $1 \mathrm{~h}$ prior to application of ATP $(10 \mu \mathrm{M})$ and control data were collected at 5 and $10 \mathrm{~min}$ prior to the administration of ATP. The statistical significance in the gel contraction assay was assessed with a repeated measure ANOVA with the Bonferroni post hoc test from data collected 10 to $70 \mathrm{~min}$ during application of ATP.
A

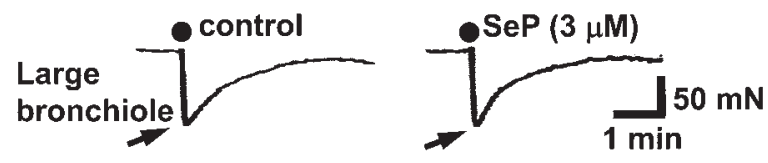

B

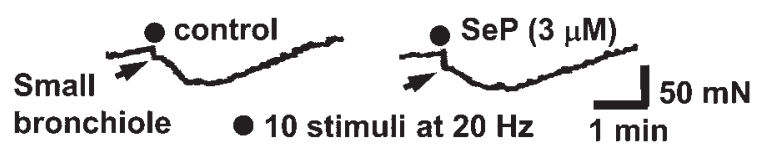

C

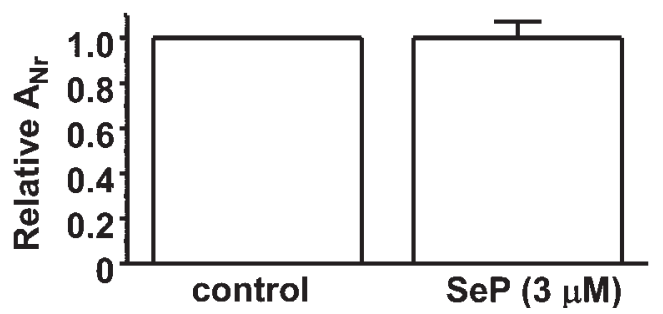

D

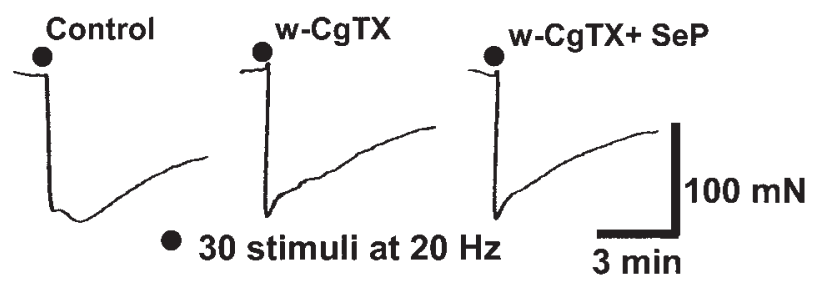

E

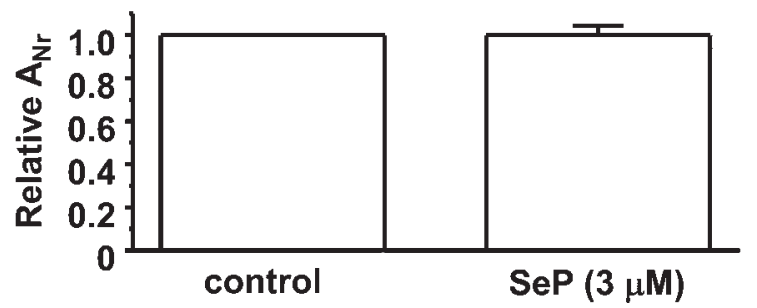

Figure 2. Effects of $\mathrm{SeP}(3 \mu \mathrm{M})$ on the NANC-relaxations evoked by EFS in the presence of atropine, guanethidine and 5-HT (10 $\mu \mathrm{M}$ each). A. SeP $(3 \mu \mathrm{M})$ showed no effect on the amplitude of monophasic NANC relaxation recorded from relatively large bronchiole. B. SeP $(3 \mu \mathrm{M})$ also showed no effect on the amplitude of biphasic NANC relaxation recorded from relatively small bronchiole. The arrowheads represent the peak amplitude of the initial fast NANC relaxation, respectively. C. Effects of $\mathrm{SeP}(3 \mu \mathrm{M})$ on the biphasic NANC relaxations recorded from relatively small bronchioles. D., E. Effects of $\operatorname{SeP}(3 \mu \mathrm{M})$ on the initial fast NANC relaxation evoked by EFS in the presence of atropine, guanethidine, $5-\mathrm{HT}$ and $\omega-\mathrm{CgTX}(2 \mu \mathrm{M})$. The first and second records show control biphasic NANC relaxation, and the initial fast component in the presence of $\omega$-CgTX, respectively. SeP showed no effect on the initial fast component of the NANC relaxation at all (third record). Relative ANr, relative amplitude of NANC relaxation. 


\section{Results}

Effects of SeP or SS on twitch-like contraction and NANC relaxation of the cat bronchiole

The ring preparation of cat bronchioles was normally quiescent and lacked spontaneous mechanical activity. Single or repetitive EFS with short pulse duration $(0.5 \mathrm{~ms})$ at high frequency $(20 \mathrm{~Hz})$ evoked TTX and atropine-sensitive twitch-like contraction, the amplitude of which was dependent on the number of stimuli at a constant stimulus frequency and intensity.

Firstly, we observed the effects of SeP and SS on the twitch-like contraction. As shown in Figure 1A and B, SeP $(3 \mu \mathrm{M})$ suppressed the amplitude of twitch like contraction evoked by EFS (10 stimuli at $20 \mathrm{~Hz}$ with $0.5 \mathrm{~ms}$ pulse duration) in the presence of quanethidine, indomethacin, L-NAME (10 $\mu \mathrm{M}$ each) and VIP-antiserum (see Materials and Methods). In addition, SS $(3 \mu \mathrm{M})$ also suppressed the amplitude of the twitch-like contraction (Fig. 1B).

In the presence of atropine and guanethidine $(1 \mu \mathrm{M}$ each), 5-hydroxytryptamine (5-HT) $(10 \mu \mathrm{M})$ increased the muscle tone, and EFS applied during the elevated muscle tone evoked mono or biphasic NANC relaxations as reported previously (Takahashi et al. 1995; Tanaka et al. 1996) (Fig. 2A,B). Namely in the relatively large $(2 \mathrm{~mm}$ O.D.) or small bronchiole (<1mm O.D.), EFS evoked mono or biphasic relaxations, respectively (Fig. $2 \mathrm{~A}, \mathrm{~B})$. SeP $(3 \mu \mathrm{M})$ did not affect the amplitude of monophasic NANC relaxation at all (Fig. 2A). However, it seemed that SeP $(3 \mu \mathrm{M})$ enhances the amplitude of the initial fast NANC relaxation in the biphasic response (Fig. 2B). To observe the effects of $\mathrm{SeP}$ on the initial fast NANC relaxation, we suppressed the generation of second component of the NANC relaxation by use of $\omega$-conotoxin GVIA ( $\omega$-CgTX GVIA). Since we have demonstrated that at least two substances, NO or NO-containing compounds for the fast relaxation and VIP or VIPlike peptide for slow relaxation are involved in the NANC relaxations (Matsuzaki et al. 1980; Palmer et al. 1986; Hakoda et al. 1991; Takahashi et al. 1995; Tanaka et al. 1996), and that N-type calcium-channel blocker $\omega$-CgTX GVIA $(1 \mu \mathrm{M})$ selectively and completely suppresses the second slow NANC relaxation without affecting the initial fast component. The initial fast component is also insensitive to P- and Q-type calcium channel blocker $\omega$-agatoxin and the L-and T-type calcium channel blocker nimodipine, thereby indicating that the initial fast NANC relaxation is due to the activation of R-type calcium channel in the pre-synaptic nerve terminal (Fujisawa et al. 1999). As shown in Figure 2D, $\omega$-CgTX GVIA abolished the second slow NANC relaxation, and the amplitude of fast NANC relaxation which was sensitive to L-NAME $(10 \mu \mathrm{M})$ or TTX $(0.1 \mu \mathrm{M})$, was not affected at all by $\operatorname{SeP}(3 \mu \mathrm{M})$ (Fig. $2 \mathrm{E})$.

\section{Effects of SeP on ATP-induced BTSMC contraction}

SeP inhibited the twitch-like contraction evoked by indirect muscle stimulation without affecting the NANC relaxation. In an attempt to study whether SeP acts on the pre-synaptic nerve terminal or on the post-synaptic airway smooth mus-
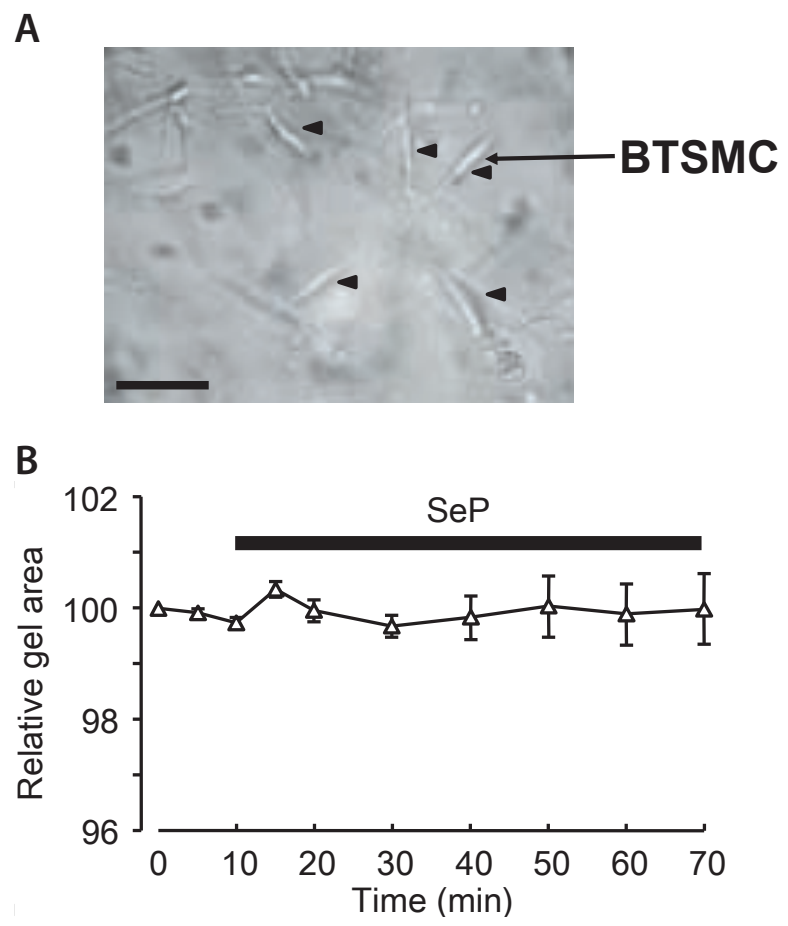

C

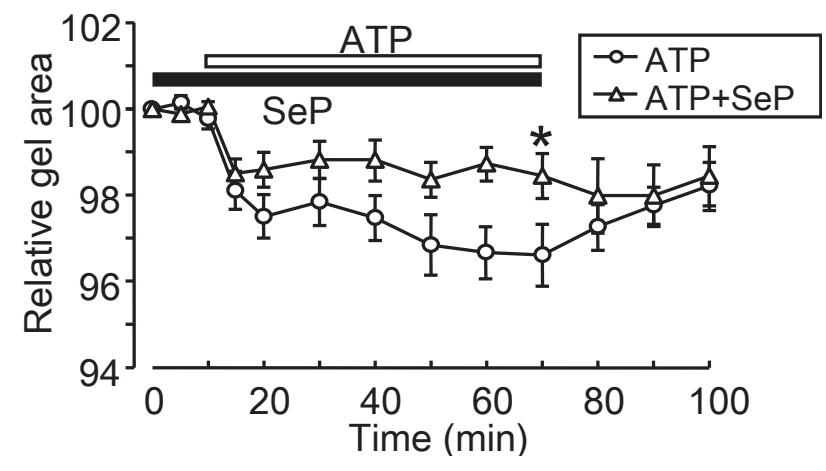

Figure 3. Effect of $\mathrm{SeP}$ on contraction of bovine tracheal smooth muscle cells (BTSMC) embedded in collagen gel. A. Microscopic view of BTSMC embedded in collagen gel. BTSMC were spindleshaped and randomly oriented in collagen gel in DIV3. Arrowheads indicate typical shape of BTSMC (magnification $\times 20$, scale: $100 \mu \mathrm{m})$. B. Effects of SeP $(3 \mu \mathrm{M})$ on contraction of gel embedded BTSMC. Relative changes of gel surface area were plotted every 5 or $10 \mathrm{~min}$. SeP was applied to the BTSMC-embedded gel at the bar. C. Effects of pretreatment with $\mathrm{SeP}(3 \mu \mathrm{M})$ on the ATP-induced gel contraction. The open bar indicates the period that $10 \mu \mathrm{M}$ ATP was applied. Each point is the average \pm S.E.M. $(n=6) ;{ }^{\star} p<0.05$. 
cle, we observed the effects of SeP on ATP-induced BTSMS contraction.

The cultured BTSMC embedded in collagen gel were spindle-shaped and randomly oriented as shown in Figure 3A. SeP $(3 \mu \mathrm{M})$ showed no effect on the surface area of the gel with embedded BTSMC at all (Fig. 3B). However, application of $10 \mu \mathrm{M}$ ATP decreased the surface area of the gel, as reported previously (Sakai et al. 2003; Ohta et al. 2008) (Fig. 3C). One hour pretreatment with SeP $(3 \mu \mathrm{M})$ significantly suppressed ATP-induced gel contraction (Fig. 3C). This result suggests that pretreatment of the gel embedded BTSMC with SeP $(3 \mu \mathrm{M})$ suppresses ATP-induced contraction of BTSMC.

\section{Discussion}

Present results show that SeP or SS suppresses the amplitude of twitch-like contractions of cat bronchiole without affecting NANC relaxations evoked by EFS. In the small bronchiole, NANC relaxations are composed of initial fast and second slow components in the cat airway. However, in trachea, bronchi and relatively large bronchiole, the two components fused into mono-phasic relaxation (Hakoda et al. 1991; Takahashi et al. 1995; Tanaka et al. 1996). The present results show that $\mathrm{SeP}(3 \mu \mathrm{M})$ did not affect the amplitude of mono-phasic NANC relaxation, thereby indicating that SeP $(3 \mu \mathrm{M})$ has no effect on the initial and second components of the NANC relaxation.

To study whether the suppressive effect of SeP on the twitch-like contraction is due to the pre- or post-synaptic actions of SeP, we observed the effects of SeP on the cultured BTSMC embedded in collagen gel, and found that SeP suppresses the ATP-induced gel contraction of BTSMC. These observations, taken together, suggest that SeP suppresses the amplitude of twitch-like contraction of cat bronchiole by acting on the bronchiolar smooth muscle.

One of the toxic symptoms of selenium is respiratory failure and the effects of SS on the mouse phrenic nervediaphragm were investigated (Lin-Shiau et al. 1989). It turned out that the SS is potent in inducing contracture of the diaphragm, which causes the reduction in the amplitude of the twitch contraction induced by the indirect stimulation and then completely abolishes it. It seems reasonable to conclude that the contracture is induced by a direct action of SS on the muscle rather than that SS enhanced transmitter release from the motor nerve terminal, since denervation, $d$ tubocurarine and TTX showed no effect on the contracture. The authors suggested that the initial binding of selenium to the sulfhydryl group of the muscle membrane, which then triggers the release of $\mathrm{Ca} 2+$ from sarcoplasmic reticulum (Lin-Shiau et al. 1989).
In the rat ventricle, on the other hand, it has been shown that selenium, through alternations of cellular thiol redox status induces a dual action on muscle contraction that can be imputed to a combined action on $\mathrm{Ca}^{2+}$ channels, $\mathrm{Ca}^{2+}$-transporters and contractile proteins. Namely, extracellular negative effects of selenium are due to a partial reduction of $\mathrm{Ca}^{2+}$ current amplitude and intracellular effects are mediated by a diminution of $\mathrm{Ca}^{2+}$ handling by intracellular organelles and by a sensitization of the contractile apparatus to $\mathrm{Ca}^{2+}$. These effects of selenium seems to occur through oxidative alternation of protein thiols, since selenium-induced changes in contractile activity and $\left[\mathrm{Ca}^{2+}\right]_{\mathrm{i}}$ were irreversible on wash but could be restored by the disulfide-reducing agent, dithiothreitol (DTT). In support of this view, both reduced glutathione and protein levels significantly decreased in cells exposed to selenium (Turan et al. 1996, 1998).

In the present study, contrary to the observations in the diaphragm muscle, SeP or SS reduced the amplitude of the twitch-like contraction without affecting the resting muscle tone and NANC relaxations. The mechanisms involved in the effect of SeP or SS yet to be clarified. However, it has been shown in heart muscle that selenium causes oxidative alterations of protein thiols, which affects $\mathrm{Ca}^{2+}$ handling by intracellular organelles and plasma membrane. Therefore, it seems reasonable to assume that similar oxidative actions of selenium on proteins which concern the $\mathrm{Ca}^{2+}$ economy in the airway smooth muscle reduced the amplitude of twitch-like contraction. In addition, $\mathrm{SeP}(3 \mu \mathrm{M})$ suppresses the biphasic inward currents evoked by acetylcholine in the rat paratracheal ganglion cells (unpublished observation by Akaike). It has been reported that the concentration of SeP in human plasma ranges between $1 \sim 10 \mathrm{mg} / \mathrm{l}$ (Andoh et al. 2005), and therefore it is also reasonable to assume that $\mathrm{SeP}$ in the plasma would affect the contraction of the airway smooth muscle and nicotinic and muscarinic currents evoked by the activation of cholinergic nerve fibers in the smooth muscle and paratracheal ganglion cells.

Asthma is driven by allergic airway inflammation and involves hyper reactivity of the airway smooth muscle and increased levels of oxidative stress. This led to the speculation that antioxidants like selenium may play important roles in preventing or treating asthma. However, it turned out that selenium intake and allergic airway inflammation are not related in a simple dose-response manner, which may explain the inconsistent results obtained from previous descriptive studies in humans (Hoffmann et al. 2007, 2008). As far as the airway smooth muscle concerned, present results indicate that $\mathrm{SeP}$, which is the major selenoproteins in the plasma, suppresses the cholinergic contractions in the airway.

The present study also indicates that human plasma would contain various biologically active substances like SeP. 
In conclusion, SeP or SS suppressed the amplitude of twitch-like contractions of cat bronchiole without affecting the NANC relaxations evoked by EFS. SeP also suppressed the ATP-induced BTSMC contraction. These results suggest that SeP suppresses the amplitude of twitch-like contraction of cat bronchiole by acting directly on the bronchiolar smooth muscle.

Acknowledgement. This work was supported by Grants-in Aid from Kumamoto Health Science University for Ogawa S. K., Ito Y., Shin M. and Akaike N.

\section{References}

Amis T. C., McKiernan B. C. (1986): Systematic identification of endobronchial anatomy during bronchoscopy in the dog. Am. J. Vet. Res. 47, 2649-2657

Andoh A., Hirashima M., Maeda H., Hata K., Inatomi O., Tsujikawa T., Sasaki M., Takahashi K., Fujiyama Y. (2005): Serum selenoprotein-P levels in patients with inflammatory bowel disease. Nutrition 21, 574-579 http://dx.doi.org/10.1016/j.nut.2004.08.025

Anundi, I., Hogberg J., Stahl A. (1982): Involvement of glutathione reductase in selenite metabolism and toxicity, studied in isolated rat hepatocytes. Arch. Toxicol. 50, 113-123 http://dx.doi.org/10.1007/BF00373393

Aviado D. M., Drimal J., Watanabe T., Lish P. M. (1975): Cardiac effects of sodium selenite. Cardiology 60, 113-120 http://dx.doi.org/10.1159/000169709

Bell R. R., Soliman M. M., Nonavinakere V. K., Hammerbeck D. M., Early J. L. (1997): Selenium and cadmium induced pulmonary functional impairment and cytotoxicity. Toxicol. Lett. 90, 107-114 http://dx.doi.org/10.1016/S0378-4274(96)03834-9

Doelman C. J., Bast A. (1990): Oxygen radicals in lung pathology. Free Radic. Biol. Med. 9, 381-400 http://dx.doi.org/10.1016/0891-5849(90)90015-B

Fujisawa K.,Onoue H., Abe K.,Ito Y. (1999): Multiple calcium channels regulate neurotransmitter release from vagus nerveterminals in the cat bronchiole. Br. J. Pharmacol. 128, 262-268 http://dx.doi.org/10.1038/sj.bjp.0702791

Ge K., Xue A., Bai J., Wang S. (1983): Keshan disease-an endemic cardiomyopathy in China. Virchows Arch. A Pathol. Anat. Histopathol. 401, 1-15 http://dx.doi.org/10.1007/BF00644785

Greene L. S. (1995): Asthma and oxidant stress: nutritional, environmental, and genetic risk factors. J. Am. Coll. Nutr. 14, 317-324

Gromer S., Eubel J. K., Lee B. L., Jacob J. (2005): Human selenoproteins at a glance. Cell Mol. Life Sci. 62, 2414-2437 http://dx.doi.org/10.1007/s00018-005-5143-y

Hakoda H., Ito Y. (1990): Modulation of cholinergic neurotransmission by the peptide VIP, VIP antiserum and VIP antagonists in dog and cat trachea. J. Physiol. 428, 133-154

Hakoda H., Xie Z. Q., Aizawa H., Inoue H., Hirata M., Ito Y. (1991): Effects of immunization against VIP on neurotransmission in cat trachea. Am. J. Physiol. 261, L341-348
Hoffmann P. R., Jourdan-Le S. C., Hoffmann F. W., Chang P. S., Bollt O., He Q., Tam E. K., Berry M. J. (2007): A role for dietary selenium and selenoproteins in allergic airway inflammation. J. Immunol. 179, 3258-3267

Hoffmann P. R. (2008): Selenium and asthma: a complex relationship. Allergy 63, 854-856 http://dx.doi.org/10.1111/j.1398-9995.2008.01676.x

Inoue T., Ito Y. (1986): Characteristics of neuro-effector transmission in the smooth muscle layer of dog bronchiole and modifications by autacoids. J. Physiol. 370, 551-565

Ito Y., Tajima K. (1981): Actions of indomethacin and prostaglandins on neuro-effector transmission in the dog trachea. J. Physiol. 319, 379-392

Lin-Shiau S. Y., Liu S. H., Fu W. M. (1989): Studies on the contracture of the mouse diaphragm induced by sodium selenite. Eur. J. Pharmacol. 167, 137-146 http://dx.doi.org/10.1016/0014-2999(89)90755-3

Matsuzaki, Y., Hamasaki Y., Said S. I. (1980): Vasoactive intestinal peptide: a possible transmitter of nonadrenergic relaxation of guinea pig airways. Science 210, 1252-1253 http://dx.doi.org/10.1126/science.6254154

Mortensen J. D., Young J. D., Stout L., Stout A., Bagley B., Schaap R. N. (1983): A numerical identification system for airways in the lung. Anat. Rec. 206, 103-114 http://dx.doi.org/10.1002/ar.1092060112

Neve J. (1991): Physiological and nutritional importance of selenium. Experientia 47, 187-193 http://dx.doi.org/10.1007/BF01945424

Nonavinakere V. K., Proctor A. S., Bell R. R., Mallory Z. Y., Early J. L. (1999): An acute intratracheal selenium study: immediate effects on respiration in guinea pigs. Toxicol. Lett. 104, 231-237 http://dx.doi.org/10.1016/S0378-4274(98)00374-9

Ohta Y., Hayashi M., Kanemaru T., Abe K., Ito Y., Oike M. (2008): Dual modulation of airway smooth muscle contraction by Th2 cytokines via matrix metalloproteinase-1 production. J. Immunol. 180, 4191-4199

Palmer J. B., Cuss F. M., Barnes P. J. (1986): VIP and PHM and their role in nonadrenergic inhibitory responses in isolated human airways. J. Appl. Physiol. 61, 1322-1328

Rayman M. P. (2000): The importance of selenium to human health. Lancet 356, 233-241 http://dx.doi.org/10.1016/S0140-6736(00)02490-9

Rotruck J. T., Pope A. L., Ganther H. E., Swanson A. B., Hafeman D. G., Hoekstra W. G. (1973): Selenium: biochemical role as a component of glutathione peroxidase. Science 179, 588-590

http://dx.doi.org/10.1126/science.179.4073.588

Saito Y., Hayashi T., Tanaka A., Watanabe Y., Suzuki M., Saito E., Takahashi K. (1999): Selenoprotein P in human plasma as an extracellular phospholipid hydroperoxide glutathione peroxidase. Isolation and enzymatic characterization of human selenoprotein p. J. Biol. Chem. 274, 2866-2871 http://dx.doi.org/10.1074/jbc.274.5.2866

Sakai J., Oike M., Hirakawa M., Ito Y. (2003): Theophylline and cAMP inhibit lysophosphatidic acid-induced hyperresponsiveness of bovine tracheal smooth muscle cells. J. Physiol. 549, 171-180 http://dx.doi.org/10.1113/jphysiol.2003.039024 
Salonen J. T., Alfthan G., Huttunen J. K., Pikkarainen J., Puska P. (1982): Association between cardiovascular death and myocardial infarction and serum selenium in a matched-pair longitudinal study. Lancet 2, 175-179 http://dx.doi.org/10.1016/S0140-6736(82)91028-5

Schrauzer G. N., White D. A., Schneider C. J. (1977): Cancer mortality correlation studies--III: statistical associations with dietary selenium intakes. Bioinorg. Chem. 7, 23-31 http://dx.doi.org/10.1016/S0006-3061(00)80126-X

Schwarz K., Foltz C. M. (1999): Selenium as an integral part of factor 3 against dietary necrotic liver degeneration. 1951 Nutrition 15, 255

Takahashi N., Tanaka H., AbdullahN., Jing L., Inoue R., Ito Y. (1995): Regional difference in the distribution of L-NAMEsensitive and -insensitive NANC relaxations in cat airway. J. Physiol. 488, 709-720

Tanaka H., Jing L., Takahashi S., Ito Y. (1996): The possible role of nitric oxide in relaxations and excitatory neuroeffector transmission in the cat airway. J. Physiol. 493, 785-791
Turan B., Desilets M., Acan L. N., Hotomaroglu O., Vannier C., Vassort G. (1996): Oxidative effects of selenite on rat ventricular contractility and Ca movements. Cardiovasc. Res. 32, 351-361 http://dx.doi.org/10.1016/0008-6363(96)00071-5

Turan B., Koc E., Hotomaroglu O., Kiziltan E., Yildirim S., Demirel E. (1998): Tissue and concentration-dependent effects of sodium selenite on muscle contraction. Biol. Trace Elem. Res. 62, 265-280 http://dx.doi.org/10.1007/BF02783976

Xie Z. Q., Hirose T., Hakoda H., Ito Y. (1991): Effects of vasoactive intestinal polypeptide antagonists on cholinergic neurotransmission in dog and cat trachea. Br. J. Pharmacol. 104, 938-944 http://dx.doi.org/10.1111/j.1476-5381.1991.tb12530.x

Young J. D., Crowley C., Tucker E. M. (1981): Haemolysis of normal and glutathione-deficient sheep erythrocytes by selenite and tellurite. Biochem. Pharmacol. 30, 2527-2530 http://dx.doi.org/10.1016/0006-2952(81)90578-5

Received: May 30, 2012

Final version accepted: July 24, 2012 